\title{
Alternative Models in Genetic Analyses of Carcass Traits Measured by Ultrasonography in Guzerá cattle: A Bayesian Approach
}

\author{
Jairo Azevedo Junior ${ }^{1}$, José C. Souza ${ }^{1}$, Robert A. Cushman ${ }^{2}$, Marco C. A. M. Bink ${ }^{3}$, Caio A. Perazza ${ }^{1}$, \\ Sarah L. Meirelles ${ }^{1} \&$ Tarcisio M. Gonçalves ${ }^{1}$ \\ ${ }^{1}$ Department of Animal Science, Federal University of Lavras, Brazil \\ ${ }^{2}$ USDA-ARS, Reproduction Research Unit, Meat Animal Research Center, Clay Center, NE 68901, United \\ States \\ ${ }^{3}$ Biometris, 6700AC, Wageningen UR, Netherlands \\ Correspondence: Jairo Azevedo Junior, Department of Animal Science, Federal University of Lavras, P.O. Box 37, \\ 37200-000, Lavras-MG, Brazil. Tel: 55-35-3829-1238. E-mail: jairo@zootecnista.com.br
}

Received: May 13, 2013 Accepted: June 16, 2013 Online Published: August 15, 2013

doi:10.5539/jas.v5n9p29 URL: http://dx.doi.org/10.5539/jas.v5n9p29

\begin{abstract}
The objective was to study alternative models for genetic analyses of carcass traits assessed by ultrasonography in Guzerá cattle. Data from 947 measurements (655 animals) of Rib-eye area (REA), rump fat thickness (RFT) and backfat thickness (BFT) were used. Finite polygenic models (FPM), infinitesimal polygenic models (IPM) and FPM combined with IPM (IPM + FPM) were empirically tested, adjusting for the effects of permanent environment, age and weight at measurement and the contemporary group. A Bayesian analysis using the computer package FlexQTL ${ }^{\mathrm{TM}}$ was adopted. The combined model adjusted to the data, allowing reliable genetic analyses of REA and BFT. For the RFT, the IPM model was the only one to have convergence and, in this case, the trait should be analyzed by a polygenic model. The presence of up to three major genes (MGs) controlling the expression of REA and two MGs for BFT was detected. The additive genetic action was over dominance to REA, and for BFT the dominance genetic action was greater. Heritability estimates, and respective standard error, adjusted for the combined model to REA were $0.15(0.00025)$ for the polygenic fraction and $0.10(0.00019)$ for the oligogenic fraction; for BFT was 0.19 (0.00027) and 0.13 (0.00025), respectively. Heritability of $0.17(0.00028)$ was estimated for RFT when the model was adjusted to IPM. There are major genes segregating within the population studied for REA and BFT traits, thus, their genetic analyses must be studied considering oligogenic effects. The major gene effects detected for a small number of genes, may possibly help to increase the reliability in detecting chromosomal regions that explain and control the phenotypic expression of these traits, facilitating research on detection and validation of molecular markers in this population.
\end{abstract}

Keywords: Bayesian inference, finite and infinitesimal polygenic models, genetic action, heritability, major genes

\section{Introduction}

The Guzerá breed has excelled in the Brazilian cattle production scenario adding significant gains in productivity, hardiness, precocity and ability to produce good quality meat. Quantitative traits such as rib-eye area, rump fat thickness, and backfat thickness have been evaluated in animal breeding programs because they are correlated with several factors related to finish quality, productivity and carcass meat cut yields (Bergen, Miller, \& Wilton, 2005; Lima Neto et al., 2009). An effort should be made to increase the knowledge about the genetic architecture of these traits before they are included in a breeding program. The number and effects of genes controlling the phenotypic expression, gene action and genetic parameters are examples of this knowledge. However, such studies are scarce, especially for Bos indicus breeds such as the Guzerá.

The infinitesimal polygenic model (IPM) was introduced by Fisher (1918), assuming that traits are determined by different alleles at many loci and that the effect of each locus is small. In 1977, the finite polygenic model (FPM) was first proposed by Thompson and Skolmick (1977). The FPM allows the inclusion of non-additive genetic effects, such as dominance and epistasis, which is difficult to do with the IPM due to methodological and computational reasons (Boer \& Hoechele, 1993). Recently, a model that combines the finite and infinitesimal polygenic effects was proposed (Bink, Uimari, Sillanpää, Janss, \& Jansen, 2002; Gonçalves, Oliveira, Bovenhuis, Bink, \& Van Arendonk, 2005), which allows separating the polygenic from the major gene effect over the 
variation of the trait analyzed and may make the estimation method of variance components more flexible, robust and appropriate (Bink et al., 2002). Bayesian methods have been proposed as an option for solving problems related to the evaluation of genetic merit in populations with complex pedigrees (Bink et al., 2002; Gonçalves et al., 2005). Therefore, the objective of the current study was to investigate alternative models to describe the genetic architecture of carcass traits evaluated by real-time ultrasonography in Guzerá cattle using a Bayesian approach.

\section{Material and Methods}

\subsection{Data Collection}

Data were derived from 655 Guzerá animals (from 67 bulls and 320 cows) born between September 2002 and June 2005. Weight and carcass ultrasound data were obtained from 17.83 \pm 4.40 -month-old animals. Average weight at the time of measurements was $391 \pm 81 \mathrm{~kg}$. Herds were from four farms in São Paulo, Brazil. Animals were managed on either: grazing only, grazing with forage supplementation or fed high energy diets. The farms are located in regions characterized by either, predominantly tropical, humid, with a prevalence of fodder plant families Brachiaria and Panicum spp and annual average rainfall between 1,300 and 1,600 $\mathrm{mm}$. All animals had free access to water and mineral supplementation throughout the experimental period. Rib-eye Area (REA, $\mathrm{n}=943$ ), rump fat thickness (RFT, $\mathrm{n}=907$ ) and backfat thickness (BFT, $\mathrm{n}=917$ ) images were obtained in living animals by positioning the transducer transversely between the $12^{\mathrm{a}}$ and $13^{\mathrm{a}}$ ribs, parallely between $12^{\mathrm{a}}$ and $13^{\mathrm{a}}$ rib and at the junction between the Biceps femoris and Gluteus medium muscles, respectively. All measurements were performed by a single experienced technician using an Aloka 500V unit with a $3.5 \mathrm{MHz} / 17.2 \mathrm{~cm}$ linear transducer (Aloka Co. Ltd - Wallingford, EUA).

The relationship matrix contained 1,042 animals, 387 composed the base population, with an additional 655 related animals having one, two or three measurements of the traits considered. Outliers detection analysis was performed and the data above and below three standard deviations were removed.For increased reliability, manipulation of the database was performed using SAS 9.1.3 procedures (SAS Institute, Cary, NC - USA).

Thirty three contemporary groups (CG) were generated by gender, year and farm of birth, feeding management and measurement date. The lowest CG contained two animals per group and the largest contained 113 animals.

\subsection{Models and Statistical Analysis}

Univariate analysis was performed for all traits (REA, RFT and BFT). Analyses were adjusted for age and weight at the time of measurement, contemporary group and permanent environmental effect for all traits. The following models were used:

1) The Infinitesimal Polygenic Model (IPM):

$$
y=X \beta+W u+e
$$

2) The Finite Polygenic Model (FPM):

$$
y=X \beta+\sum_{K}^{N_{G E P}} Z_{M G} \alpha_{M G_{K}}+e
$$

3) The combined model (IPM+FPM) adjusted for each trait analyzed was:

$$
y=X \beta+W u+\sum_{K}^{N_{G E P}} Z_{M G} \alpha_{M G_{K}}+e
$$

In the three models: $\boldsymbol{y}=$ is the vector of observations; $\boldsymbol{X}=$ incidence matrix of non-genetic factors, which connects the phenotypes of non-genetic effects; $\boldsymbol{\beta}=$ is a vector containing the mean $(\mu)$ and all non-genetic factors affecting the traits of interest: animal age and body weight at the time of measurement, contemporary group (sex, birth year, farm of birth, feed management and date of measurement) and permanent environmental effect (Lima Neto et al., 2009); $\boldsymbol{W}=$ incidence matrix of the genetic random direct effects; $\boldsymbol{u}=$ is the vector of random effects of direct genetic values; the effects of several genes with infinitesimal effects that are not explained by the major gene are computed here; $\boldsymbol{Z}_{\boldsymbol{M G}}=$ incidence matrix that connects the phenotypic information to MG is typically unknown, because the genotypes of individuals are not known. However, this matrix can be inferred from the pedigree and phenotype (segregation indicators) (Bink et al., 2002) and thus, molecular data were not used. It is assumed that the MG is biallelic, allowing three different genotypes (AA, Aa and aa) and having genotypic values equal to $+\mathbf{a}, \mathbf{d}$ and - a, respectively. The variables $\alpha$ and $\delta$ represent the additive and dominance effects of a single gene. The matrix $\mathbf{Z}$ size depends on the number of MGs in the model; $N_{M G}=$ is the number of major genes. This number is considered a random variable and makes inferences about the data distribution; $\boldsymbol{\alpha}_{\boldsymbol{M G}}=$ is a bidimensional vector for the $\mathrm{k}^{\text {th }}$ major gene (MG), i.e., additive (a) and dominance (d) effects were adjusted here; $\boldsymbol{e}=$ vector of the normally distributed residual associated with each observation. After some preliminary analyses, in these models the presence of a maximum of fifteen and at least one locus for major genes (MGs), and one locus of infinitesimal 
polygenic effect were assumed to explain the genetic variation found. These assumptions are necessary to decrease the computational statistical requisites and gives an informative priori information to the Bayesian inferences.

In summary, the assumptions are: in order to use the IPM only polygenic effects are responsible for the trait expression (Fisher, 1918); for the FPM only the oligogenic effects control the trait expression (Thompson and Skolmick, 1977; Boer \& Hoechele, 1993) and for the IPM+FPM, both effects can be observed (Bink et al., 2002; Gonçalves et al., 2005).

\subsection{Bayesian Inferences}

A Bayesian approach to the analyses was adopted. The Gibbs Sampling and Markov Chain Monte Carlo (MCMC) methods were used to update the parameters considered in the models. Thus, on each MCMC analysis, considering a single Markov chain, by Gibbs sampler and the reversible jump sampler (Metropolis-Hastings), the number of sampling cycles was $1,500,000$. At every 20 cycles, a sample was saved. The discard sampling period, e.g., number of cycles that must be discarded before samples produced by the Gibbs sampler can be regarded as samples from the posterior distribution (burn-in) was 7,500 cycles (samples). Therefore, 67,500 samples were used to analyze posteriori parameter distributions. To perform this Bayesian analysis, the software FlexQTL ${ }^{\mathrm{TM}}$ (Bink, M.C.A.M., Version 0.98, Wageningen, 2009) was used, according to the studies of Uimari and Sillanpää (2001).

For non-genetic variables, such as the animal age and animal weight at the time of measurement, contemporary group, and permanent environment, a Normal prior was assumed. The variance of this normal distribution is unknown and follows a chi-square $\left(\chi^{2}\right)$ inverted staggered distribution (Sorensen \& Gianola, 2002). In the case of general average, the mean of Normal distribution was dependent on the data:

$$
\hat{\mu}=y=1 / n \sum_{i}^{n} y_{i}
$$

The prior distribution for the residual variance follows a chi-square $\left(\chi^{2}\right)$ inverted staggered. As $A$ is the matrix of additive genetic relationship, given the knowledge of the animal pedigree, then the prior distribution for polygenic effects can be given by:

$$
I P M \mid A \sigma_{I P M}^{2} \sim N\left(0, A \sigma_{I P M}^{2}\right)
$$

where $\sigma_{I P M}^{2}$ is the polygenic genetic variance with chi-square $\left(\chi^{2}\right)$ inverted staggered distribution.

The MGs additive and dominance effects follow a univariate normal distribution, whose variance is dependent on the data set and the number of MGs in the model, as proposed by Yi (2004) and Yi et al. (2005).

Considering $\hat{\sigma}_{y}^{2}$ as the estimate of trait phenotypic variance:

$$
\hat{\sigma}_{y}^{2}=1 / n \sum_{i}^{n}\left(y_{i}-\bar{y}\right)^{2}
$$

then:

where,

$$
p(\alpha) \sim N\left(0, \sigma_{\alpha\left(N_{M G}\right)}^{2}\right)
$$

$$
\sigma_{\alpha\left(N_{M G}\right)}^{2}=\sigma_{\alpha}^{2} / N_{M G}
$$

and

$$
\sigma_{\alpha}^{2} /\left(2 x \hat{\sigma}_{y}^{2}\right) \sim \beta(2,30)
$$

considering the biallelic effect of gene. This implies that the variance of the normal distribution decreases as the number of genes in the model increases and vice-versa.

The normal distribution was assumed for the conditional distribution of the observations (Y), and, when combined with the prior distributions, the joint posterior distribution of all interest parameters was obtained. Once this joint distribution has no standard form known and is computationally intractable, marginal conditional distribution samples were generated by the Monte Carlo via Markov Chain (MCMC) methods (Bink et al., 2002; Gelman, Carlin, Stern, \& Rubin, 2004). In MCMC simulation, the Gibbs sampler was used to obtain marginal distribution samples of random variables. The number of MGs $\left(N_{M G}\right)$ was added as a unknown variable by the algorithm "Reversible Jump Sampler (Metropolis-Hastings)" described by Green (1995) and Bink et al. (2002).

Chain convergence was diagnosed when the effective chain size (ECS) reached a number greater or equal to 100 (Sorensen, Andersen, \& Gianola, 1995). The detection of a major gene affecting the expression traits was based on posteriori estimations for the number of MGs $\left(N_{M G}\right)$, on posteriori probability for MGs and on the Bayes factor (Kass \& Raftery, 1995). 


\subsection{Heritability}

Inferences about the posteriori heritabilitiy distributions were obtained based on Gibbs sampling of variance components (posterior distributions). The phenotypic variance were decomposed in polygenic variance $\left(\sigma_{I P M}^{2}\right)$, oligogenic $\left(\sigma_{M G}^{2}\right)$, additive genetic $\left(\sigma_{a}^{2}\right)$, permanent environmental $\left(\sigma_{P E}^{2}\right)$, age at measurement $\left(\sigma_{A G E}^{2}\right)$, body weight at measurement $\left(\sigma_{B W}^{2}\right)$, contemporary group $\left(\sigma_{C G}^{2}\right)$ and error $\left(\sigma_{r}^{2}\right)$, whose were used to the heritability estimates. For model 1 (IPM):

$$
h_{I P M}^{2}=\sigma_{I P M}^{2} \cdot\left(\sigma_{I P M}^{2}+\sigma_{P E}^{2}+\sigma_{A G E}^{2}+\sigma_{B W}^{2}+\sigma_{C G}^{2}+\sigma_{r}^{2}\right)^{-1}
$$

for model 2 (FPM):

$$
h_{F P M}^{2}=\sigma_{a}^{2} \cdot\left(\sigma_{M G}^{2}+\sigma_{P E}^{2}+\sigma_{A G E}^{2}+\sigma_{B W}^{2}+\sigma_{C G}^{2}+\sigma_{r}^{2}\right)^{-1}
$$

and for model 3 (IPM + FPM) the equations:

$$
h_{I P M}^{2}=\sigma_{I P M}^{2} \cdot\left(\sigma_{I P M}^{2}+\sigma_{M G}^{2}+\sigma_{P E}^{2}+\sigma_{A G E}^{2}+\sigma_{B W}^{2}+\sigma_{C G}^{2}+\sigma_{r}^{2}\right)^{-1}
$$

and

$$
h_{F P M}^{2}=\sigma_{a}^{2} \cdot\left(\sigma_{I P M}^{2}+\sigma_{M G}^{2}+\sigma_{P E}^{2}+\sigma_{A G E}^{2}+\sigma_{B W}^{2}+\sigma_{C G}^{2}+\sigma_{r}^{2}\right)^{-1}
$$

Thus, the phenotypic variance $\left(\sigma_{P}^{2}\right)$ is the sum of the denominator of each one of these equations.

\section{Results and Discussion}

The phenotypic means for rib-eye area (REA), rump fat thickness (RFT) and backfat thickness (BFT) were 58.17 $\mathrm{cm}^{2}, 2.36 \mathrm{~mm}$ and $3.36 \mathrm{~mm}$, respectively. Considering the average age at measurements of animals, these phenotypic means are in agreement with those of previously published reports (Vittori et al., 2006; Faria, Magnabosco, Albuquerque, Bezerra, \& Lobo, 2009). For RFT, these animals did not reach a slaughter fat thickness minimum of $3 \mathrm{~mm}$.

Variance components for REA, RFT and BFT and their effective chain sizes (ECS) adjusted by the different models (IPM, FPM and IPM + FPM), are presented in Table 1. Except for RFT, the ECS of the variance components was greater than 100 . Thus, the chains converged, providing reliable inferences of variance components when these models were adjusted for these traits. Rump fat thickness has yet to be analyzed genetically through the infinitesimal polygenic model, because the finite polygenic model and the combined model

\begin{tabular}{|c|c|c|c|c|}
\hline \multirow[t]{2}{*}{ Trait $^{2}$} & \multirow[t]{2}{*}{ Model $^{3}$} & \multicolumn{3}{|c|}{ Variance Components $^{1}$} \\
\hline & & $\sigma_{I P M}^{2}(E C S)$ & $\sigma_{M G}^{2}(E C S)$ & $\sigma_{r}^{2}(E C S)$ \\
\hline \multirow[t]{3}{*}{ REA } & IPM & $36.53(3,144)$ & & $21.36(5,804)$ \\
\hline & FPM & & $35.32(1,521)$ & $21.10(889)$ \\
\hline & IPM + FPM & $23.14(204)$ & $29.57(564)$ & $10.56(549)$ \\
\hline \multirow[t]{3}{*}{ RFT } & IPM & $0.12(2,782)$ & & $0.28(3,965)$ \\
\hline & FPM & & $0.42(9)$ & $<0.00(26)$ \\
\hline & IPM + FPM & $0.02(185)$ & $0.30(538)$ & $0.08(96)$ \\
\hline \multirow[t]{3}{*}{ BFT } & IPM & $0.62(8,317)$ & & $0.67(9,272)$ \\
\hline & FPM & & $0.91(242)$ & $0.33(159)$ \\
\hline & IPM + FPM & $0.42(142)$ & $0.72(323)$ & $0.13(145)$ \\
\hline
\end{tabular}
adjusted did not reach chain convergence for all parameters in the model. This result did not allow inferences on RFT parameters and implies in further investigation about this trait.

Table 1. Variance components and their respective effective chain sizes (ECS) after adjusting the tested models

${ }^{1}$ Polygenic variance $\left(\sigma_{I P M}^{2}\right)$, oligogenic variance $\left(\sigma_{M G}^{2}\right)$ and residual variance $\left(\sigma_{r}^{2}\right) \cdot{ }^{2}$ Rib- eye area (REA), rump fat thickness (RFT) and backfat thickness (BFT). ${ }^{3}$ Infinitesimal polygenic model (IPM), finite polygenic model (FPM), and FPM combined with IPM (IPM + FPM).

Assuming that the combined model is the most suitable for genetic analyses of REA and BFT, as mentioned previously, the posteriori probabilities of major genes for these traits were calculated and presented on Table 2 . The major probabilities were located between two and three MGs for REA and a large probability of two major 
genes for BFT. When Bayes factors are considered (Table 3), there is evidence that three MGs control the expression of REA and decisive evidence for two MGs for BFT.

The posteriori estimative of major gene numbers $\left(\mathbf{N}_{\mathbf{M G}}\right)$ affecting REA and BFT expression, adjusted by the combined model, are illustrated on Table 4, and indicate up to three MGs responsible for the variation of REA and two MGs for BFT, suggesting that major genes are being segregated in this population. Furthermore, these findings imply that these traits must be evaluated genetically considering the presence of polygenes and oligogenes controlling their expression.

Table 2. Posteriori probabilities, adjusted with the combined model, for major genes number (MG) affecting carcass traits

\begin{tabular}{llllllllllll}
\hline MG & $\mathbf{0}$ & $\mathbf{1}$ & $\mathbf{2}$ & $\mathbf{3}$ & $\mathbf{4}$ & $\mathbf{5}$ & $\mathbf{6}$ & $\mathbf{7}$ & $\mathbf{8}$ & $\mathbf{9} \ldots \mathbf{1 4}$ & $\mathbf{1 5}$ \\
\hline Priori & $\mathbf{0 . 3 6 8}$ & $\mathbf{0 . 3 6 8}$ & $\mathbf{0 . 1 8 4}$ & $\mathbf{0 . 0 6 1}$ & $\mathbf{0 . 0 1 5}$ & $\mathbf{0 . 0 0 3}$ & $\mathbf{0 . 0 0 1}$ & $\mathbf{0}$ & $\mathbf{0}$ & $\mathbf{0}$ & $\mathbf{0}$ \\
\hline Trait $^{1}$ & \multicolumn{8}{c}{ Posteriori Probabilities } \\
\cline { 2 - 10 } REA & 0.001 & 0.092 & 0.332 & 0.304 & 0.163 & 0.065 & 0.026 & 0.005 & 0.002 & 0.003 & 0.007 \\
BFT & 0 & 0.002 & 0.889 & 0.091 & 0.013 & 0.002 & 0 & 0 & 0 & 0 & 0.003 \\
\hline
\end{tabular}

${ }^{1}$ Rib-eye area (REA) and backfat thickness (BFT).

Table 3. Bayes Factor ${ }^{1}$, adjusted with the combined model, for major gene numbers affecting carcass traits

\begin{tabular}{llllllllc}
\hline & \multicolumn{8}{c}{ Evidence $^{2}$} \\
\cline { 2 - 9 } Trait $^{3}$ & $\mathbf{1 / 0}$ & $\mathbf{2 / 1}$ & $\mathbf{3 / 2}$ & $\mathbf{4 / 3}$ & $\mathbf{5 / 4}$ & $\mathbf{6 / 5}$ & $\mathbf{7 / 6}$ & $\mathbf{8 / 7} . . \mathbf{1 5} / \mathbf{1 4}$ \\
\cline { 2 - 9 } REA & 8.6 & 4.0 & 2.0 & 1.5 & 1.4 & 1.7 & 0.7 & NA \\
BFT & NA & 14.0 & -2.4 & -1.1 & -2.9 & NA & NA & NA \\
\hline
\end{tabular}

${ }^{1}$ Twice the natural logarithm (2ln) of the Bayes factor is similar to the statistical test because of the likelihood. ${ }^{2}$ Evidence of $1 \mathrm{MG}$ in the model versus no or $0 \mathrm{MG}$ in the model: 0 to $2=$ low, 2 to $5=$ positive, 5 to $10=$ strong, $>10=$ decisive; $\mathrm{NA}=$ not available due to lack of MCMC samples. Negative values indicate evidence of the MGs number indicated by the denominator of the ratio. ${ }^{3}$ Rib-eye area (REA) and backfat thickness (BFT).

Table 4. Posteriori means to genetic parameters of carcass traits

\begin{tabular}{lcccccccccc}
\hline & \multicolumn{10}{c}{ Parameters $^{1}$} \\
\cline { 2 - 11 } Trait & $\boldsymbol{N}_{\boldsymbol{M G}}$ & $\boldsymbol{a}$ & $\boldsymbol{d}$ & $\sigma_{a}^{2}$ & $\sigma_{d}^{2}$ & $\sigma_{M G}^{2}$ & $\sigma_{I P M}^{2}$ & $\sigma_{P}^{2}$ & $h_{I P M}^{2}(\boldsymbol{S E})$ & $h_{F P M}^{2}(\boldsymbol{S E})$ \\
\hline REA & 3 & 9.93 & -7.45 & 15.78 & 12.03 & 29.57 & 23.14 & 156.71 & $0.15(0.00025)$ & $0.10(0.00019)$ \\
BFT & 2 & 1.97 & -2.19 & 0.30 & 0.43 & 0.72 & 0.42 & 2.23 & $0.19(0.00027)$ & $0.13(0.00025)$ \\
RFT & & & & & & & 0.12 & 0.71 & $0.17(0.00028)$ &
\end{tabular}

${ }^{1}$ Parameters adjusted with the combined model (IPM + FPM) to the rib-eye area (REA) and backfat thickness (BFT) and the infinitesimal polygenic model (IPM) adjusted to the rump fat thickness (RFT). Number of major genes $\left(N_{M G}\right)$; additive effect $(a)$; dominance effect $(d)$; additive variance $\left(\sigma_{a}^{2}\right)$; dominance variance $\left(\sigma_{d}^{2}\right)$; major gene variance $\left(\sigma_{M G}^{2}\right)$; polygenic variance $\left(\sigma_{I P M}^{2}\right)$; phenotypic variance $\left(\sigma_{P}^{2}\right)$; polygenic $\left(h_{I P M}^{2}\right)$ and oligogenic heritability $\left(h_{F P M}^{2}\right)$ and respective standard errors (SE).

Results from the Bovine 50K BeadChip clearly demonstrate the difficulties associated with polygenic effects when looking at large-scale genotyping for quantitative traits such as growth (Snelling et al., 2010) and reproduction (Fortes et al., 2010). The major gene effects detected for a small number of genes may possibly help to increase the reliability in detecting chromosomal regions that explain and control the phenotypic expression of these traits, facilitating research on detection and validation of molecular markers in Guzerá cattle. If we can focus on genes with major effects, identify those genes, and validate them in a number of populations, then, marker assisted selection may be an effective tool for improving carcass traits in Guzerá cattle. Still, we must be careful with this 
approach, because the use of markers for carcass traits could have detrimental effects on other production traits. For example, a single nucleotide polymorphism in the Titin gene has been associated with marbling in Japanese Black cattle (Yamada et al., 2009); however, a series of markers in and around Titin have been associated with heifer fertility traits (R. A. Cushman, USDA-ARS-NPA-MARC, Reproduction Research Unit, Clay Center, United States, personal communication).

A partial dominance gene action $(|d|<|a|)$ was observed for REA and an overdominance gene action $(|d|>|a|)$ was observed for BFT (Table 4). Negative values were observed for the genotypic value $d$. A possible explanation for these negative values is that dominance type actions are associated with intralocus allelic interactions, and these interactions may cause the value of the heterozygote genotype to contribute negatively to the phenotypic expression of the trait. In crossbreeding, this effect can reduce the backfat thickness of the progeny.

Heritability estimations for REA were 0.15 for the polygenic and 0.10 for the oligogenic fractions. These values were lower than 0.19 and 0.13 observed for BFT, respectively. The low heritabilities found for REA and BFT are linked to a low response to selection. For RFT, as the model IPM was the only one to fit properly, the heritability calculated by this model was 0.17 (Table 4).

Similar heritabilities were founded for REA, RFT, and BFT for taurine cattle (Kemp, Herring \& Kaiser, 2002; Reverter, Johnston, Graser, Wolcott \& Upton, 2000; Robinson, Hammond \& Mcdonald, 1993; Pitchford et al., 2006). In a study with Guzerá cattle, heritabilities were estimated, by uni/multi-trait models, respectively, of 0.35 $(0.09)$ and $0.34(0.09)$ for REA, $0.20(0.08)$ and $0.32(0.02)$ for BFT and $0.05(0.06)$ and $0.10(0.08)$ for RFT (Lima Neto et al., 2009). This shows that with the use of Bayesian analysis, the oligogenic and polygenic effects can be distinguished, and the sum of these effects for each trait is in agreement with that obtained by classical analysis.

As discussed earlier, for REA and BFT, for which major genes were detected, marker-assisted selection can be justified. However, there was also evident polygenic effect explaining part of the variation, justifying the simultaneous use of the traditional selection method (classic) based on infinitesimal polygenic effect. Thus, better responses to selection would be obtained. The lack of chain convergence justifies the use of the classical selection method to improve RFT.

\section{Conclusions}

Results from the present study demonstrate that REA and BFT are subject to polygenic and oligogenic effects. Genetic analyses of these traits should be performed with a model that combines the oligogenic and polygenic effects, justified by the efficiency of IPM+FPM model in describing the genetic architecture of these traits. These major genes could be targets for developing genetic markers; however, caution is required to ensure that such markers and the relationship between traits and selection strategy do not negatively affect other production traits such as fertility. Due to partial dominance for REA and over dominance for BFT, better results in breeding programs should be achieved when animals are subjected to selection and mating, respectively. Moreover, the breeder must pay attention to crossbreeding between heterozygous animals, because their genotype values may contribute negatively to the phenotypic expression of BFT.

\section{Acknowledgements}

The authors gratefully acknowledge financial assistance provided by the Conselho Nacional de Desenvolvimento Científico e Tecnológico - CNPq and the Fundação de Amparo à Pesquisa do Estado de Minas Gerais FAPEMIG.

\section{References}

Bergen, R., Miller, S. P., \& Wilton, J. W. (2005). Genetic correlations among indicator traits for carcass composition measured in yearling beef bulls and finished feedlot steers. Canadian Journal of Animal Science, 85, 463-473. http://dx.doi.org/10.4141/A05-013

Bink, M. C. A. M., Uimari, P., Sillanpää, M. J., Janss, L. L. G., \& Jansen, R. C. (2002). Multiple QTL mapping in related plant populations via a pedigree analysis approach. Theoretical and Applied Genetics, 104(5), 751-762. http://dx.doi.org/10.1007/s00122-001-0796-x

Boer, I. J. M., \& Hoechele, I. (1993). Genetic evaluation methods for populations with dominance and inbreeding. Theoretical and Applied Genetics, 86(2/3), 245-258.

Faria, C. U., Magnabosco, C. D., Albuquerque, L. G., Bezerra, L. A. F., \& Lobo, R. B. (2009). Genetic correlation estimates between visual scores and carcass traits measured by ultrasound in Nelore cattle using linear-threshold Bayesian models. Revista. Brasileira de Zootecnia, 38(11), 2144-2151. http://dx.doi.org/10.1590/S1516-35982009001100011 
Fisher, R. A. (1918). The correlation between relatives on the supposition of Mendelian inheritance. Transactions of the Royal Society of Edinburgh: Earth and Environmental Science, Edinburgh, 52(1), 399-433.

Fortes, M. R., Reverter, A., Zhang, Y., Collis, E., Nagaraj, S. H., Jonsson, N. N., ... Hawken, R. J. (2010). Association weight matrix for the genetic dissection of puberty in beef cattle. Proceedings of the National Academy of Sciences, USA, 107, 13642-13647. http://dx.doi.org/10.1073/pnas.1002044107

Gelman, A., Carlin, J. B., Stern, H. S., \& Rubin, D. B. (2004). Bayesian data analysis (2nd ed.). Boca Raton: Chapman \& Hall.

Gonçalves, T. M., Oliveira, H. N., Bovenhuis, H., Bink, M. C. A. M., \& Van Arendonk, J. (2005). Comparison of different strategies to analyze growth and carcass traits in a crossbred pig population: Finite and Infinitesimal Polygenic Models. Revista Brasileira de Zootecnia, 34(5), 1531-1539. http://dx.doi.org/10.1590/S1516-35982005000500013

Green, P. J. Dec. (1995). Reversible jumping Markov chain Monte Carlo computation and Bayesian model determination. Biometrika, 82(4), 711-732. New York. http://dx.doi.org/10.1093/biomet/82.4.711

Kass, R. E., \& Raftery, A. E. (1995). Bayes factors. Journal of the American Statistical Association, 90(430), 773-795. New York . http://dx.doi.org/10.1080/01621459.1995.10476572

Kemp, D. J., Herring, W. O., \& Kaiser, C. J. (2002). Genetic and environmental parameters for steer ultrasound and carcass traits. Journal of Animal Science, 80(6), 1489-1496. Champaign.

Lima Neto, H. R., Bergmann, J. A. G., Gonçalves, T. M., Araújo, F. R. C., Bezerra, L .A. F., Saiz, R. D., ... Silva, M. A. (2009). Genetic parameters for body weight and real-time ultrasound carcass traits of Guzera cattle. Arquivo Brasileiro de Medicina Veterinária e Zootecnia, 61(1), 251-258. http://dx.doi.org/10.1590/S0102-09352009000100035

Pitchford, W. S., Mirzaei, H. M., Deland, M. P .B., Afolayan, R. A., Rutley, D. L., \& Verbyla, A. P. (2006). Variance components for birth and carcass traits of crossbred cattle. Australian Journal of Experimental Agriculture, 46(2), 225-231. Melbourne .http://dx.doi.org/10.1071/EA05248

Reverter, A., Johnston, D. J., Graser, H. U., Wolcott, M. L., \& Upton, W. H. (2000). Genetic analyses of live animal ultrasound and abattoir carcass traits in Australian Angus and Hereford cattle. Journal of Animal Science, 78(7), 1786-1795.

Robinson, D. L., Hammond, K., \& Mcdonald, C. A. (1993). Live animal measurement of carcass traits: estimation of genetic parameters for beef cattle. Journal of Animal Science, 71(5), 1128-1135.

Snelling, W. M., Allan, M. F., Keele, J. W., Kuehn, L. A., McDaneld, T., Smith, T. P. L., .. Bennett, G. L. (2010). Genome-wide association study of growth in crossbred beef cattle. Journal of Animal Science, 88, 837-848. http://dx.doi.org/10.2527/jas.2009-2257

Sorensen, D. A., Andersen, S., \& Gianola, D. (1995). Bayesian inference in threshold models using Gibbs sampling. Genetics Selection Evolution, 27(3), 229-249. Paris. http://dx.doi.org/10.1186/1297-9686-27-3-229

Sorensen, D. A., \& Gianola, D. (2002). Likelihood, Bayesian, and MCMC methods in quantitative genetics. New York: Springer-Verlag.

Thompson, E. A., \& Skolmick, M. H. (1977). Likelihoods on complex pedigrees for quantitative traits. In E. Pollack, O. Kempthorne, \& T. B. Boiley Junior (Eds.), Proceedings of international conference on quantitative genetics (pp. 815-818). Ames: Iowa State University.

Uimari, P., \& Sillanpää, M. A. (2001). Bayesian MCMC linkage analysis with segregation indicators for complex pedigrees. Genetic epidemiology, 21(3), 224-242. New York. http://dx.doi.org/10.1002/gepi.1031

Vittori, A., Queiroz, A. C., Resende, F. D., Gesualdi Junior, A., Alleoni, G. F., Razook, A. G., ... Gesualdi, A. C. L. S. (2006). Carcass traits of finishing steers and bulls from different genetic groups. Revista Brasileira de Zootecnia, 35, 2085-2092. http://dx.doi.org/10.1590/S1516-35982006000700028

Yamada, T., Sasaki, S., Sukegawa, S., Yoshioka, S., Takahagi, Y., Morita, M., ... Sasaki, Y. (2009). Association of a single nucleotide polymorphism in titin gene with marbling in Japanese Black beef cattle. Biomed Central Research Notes, 2, 78. http://dx.doi.org/10.1186/1756-0500-2-78

Yi, N. J. (2004). A unified Markov chain Monte Carlo framework for mapping multiple quantitative trait loci. Genetics, 167(2), 967-975. Berlin. http://dx.doi.org/10.1534/genetics.104.026286 
Yi, N. J., Yandell, B. S., Churchill, G. A., Allison, D. B., Eisen, E. J., Pomp, D. (2005). Bayesian model selection for genome-wide epistatic quantitative trait loci analysis. Genetics, 170(3), 1333-1344. Berlin. http://dx.doi.org/10.1534/genetics.104.040386

\section{Copyrights}

Copyright for this article is retained by the author(s), with first publication rights granted to the journal.

This is an open-access article distributed under the terms and conditions of the Creative Commons Attribution license (http://creativecommons.org/licenses/by/3.0/). 